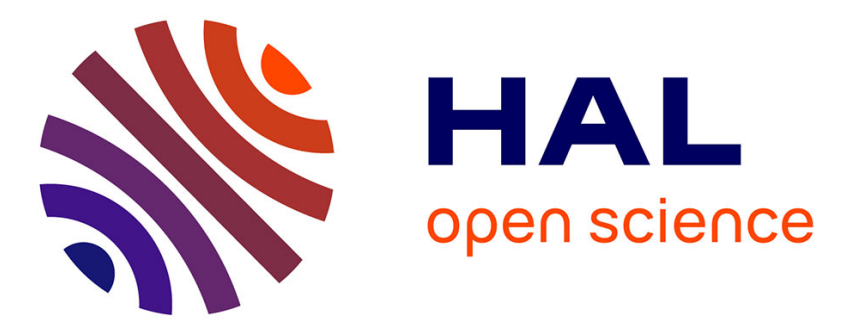

\title{
RECONSTRUCTION DES PARAMÈTRES ACOUSTIQUES D'UN FOND MARIN STRATIFIÉ À PARTIR DE SON COEFFICIENT DE RÉFLEXION
}

\author{
M. Lambert, R. de Oliveira Bohbot, D. Lesselier
}

\section{- To cite this version:}

M. Lambert, R. de Oliveira Bohbot, D. Lesselier. RECONSTRUCTION DES PARAMÈTRES ACOUSTIQUES D'UN FOND MARIN STRATIFIÉ À PARTIR DE SON COEFFICIENT DE RÉFLEXION. Journal de Physique IV Proceedings, 1992, 02 (C1), pp.C1-945-C1-948. 10.1051/jp4:19921206 . jpa-00251172

HAL Id: jpa-00251172

https://hal.science/jpa-00251172

Submitted on 1 Jan 1992

HAL is a multi-disciplinary open access archive for the deposit and dissemination of scientific research documents, whether they are published or not. The documents may come from teaching and research institutions in France or abroad, or from public or private research centers.
L'archive ouverte pluridisciplinaire HAL, est destinée au dépôt et à la diffusion de documents scientifiques de niveau recherche, publiés ou non, émanant des établissements d'enseignement et de recherche français ou étrangers, des laboratoires publics ou privés. 


\title{
RECONSTRUCTION DES PARAMĖTRES ACOUSTIQUES D'UN FOND MARIN STRATIFIÉ À PARTIR DE SON COEFFICIENT DE RÉFLEXION
}

\author{
M. LAMBERT, R. de OLIVEIRA BOHBOT et D. LESSELIER \\ Equipe Electromagnétisme, Laboratoire des Signaux et Systèmes, CNRS-ESE, F-91192 Gif-sur-Yvette \\ cedex, France
}

\begin{abstract}
Résumé : Le fond marin est représenté par une couche fluide inhomogène reposant sur un demiespace fluide homogène. Ses paramètres acoustiques (vitesse $c$ des ondes de compression, densite $\rho)$ ne dépendent que de la profondeur $z$. Pour une insonation plane à incidence donnée $\theta$, l'introduction d'une profondeur apparente $s$ fonction de la densité fait apparaître un indice $N_{\theta}(s)$ dont la connaissance à deux incidences conduit aux profils du fond $c(z)$ et $\rho(z)$. Le calcul de $N_{\theta}$ à partir du coefficient de réflexion (problème inverse non linéaire) se mène à l'aide de deux méthodes. La première est du type Born-distordue itératif et implique la résolution d'une succession de problèmes inverses linéaires. La seconde, développée à l'origine pour le diagnostic d'une permittivité électrique à moyens contrastes, fournit un accès direct à un $N_{\theta}$ approché.
\end{abstract}

\begin{abstract}
The seabed is modeled as a fluid inhomogeneous layer above a homogeneous fluid halfspace. Acoustic parameters (P-wave velocity $c$, density $\rho$ ) depend on depth $z$ only. In case of a plane insonification at given incidence $\theta$, introducing an apparent depth $s$ function of the density leads to a refractive index $N_{\theta}(s)$ whose knowledge at two incidences yields seabed profiles $c(z)$ and $\rho(z)$. Calculation of $N_{\theta}$ from the reflection coefficient (non linear inverse problem) is carried out by two methods. The first one is a distorted-Born iterative method and implies solving a succession of linear inverse problems. The second, developed earlier for probing electrical permittivity with median contrasts, directly provides an approximate profile $N_{\theta}$.
\end{abstract}

\section{Introduction}

Cette recherche porte sur le diagnostic des paramètres acoustiques du fond marin à partir de la connaissance des ondes sonores que ce dernier réfléchit quand on le soumet à une excitation connue. Rappelons en effet que les paramètres acoustiques du fond jouent un rôle prépondérant dans la propagation des ondes sonores lorsqu'il est à faible profondeur [1] et que les difficultés de mesures à partir d'échantillons prélevés sont réelles. D'où l'intérêt à priori d'un tel diagnostic et cela même quand le problème de référence est, comme ici, la très étudiée reconstruction de milieux 1-D. Cette recherche a déjà fourni certains résultats. Le lecteur se référera au résumé [2] et à une présentation préliminaire de la méthode de Born-distordue [3].

\section{Modélisation}

Le modèle est celui de la figure 1. Le fond est assimile à une couche horizontale inhomogène (un sédiment) reposant sur un socle homogène semi-infini. Les milieux sont des fluides linéaires et isotropes de densité $\rho$. Seules des ondes de compression sont supportées, de célérité $c$. Par construction $c$ et $\rho$ ne dépendent que de la profondeur $z$ et sont constantes au delà d'un certain $d$ qui, de fait, détermine la position de l'interface sédiment/socle. Les pertes par absorption, s'il y en a, varient linéairement avec la fréquence et interviennent dans la constante de propagation $k=\omega^{\prime} c+j \omega \alpha$ (dépendance des champs en $e^{-j \omega t}$ ). Ce fond est insoné sous incidence $\theta$ par une onde plane générée dans l'eau de paramètres $c_{0}$ et $\rho_{0}$ et de constante de propagation $k_{0}\left(\lambda_{0}=2 \pi / k_{0}\right)$. Il est facile de montrer que la pression acoustique $p(x, z)$ à fréquence $\omega$ et en tout point du milieu s'écrit $p(z) \exp \left(j k_{0} x \cos \theta\right)$ et est telle que $p(z)$ satisfait l'équation d'onde traditionnelle $p^{\prime \prime}(z)+\left[k_{0} k_{\theta}(z)\right]^{2} p(z)=[\log \rho(z)]^{\prime} p^{\prime}(z), k_{\theta}^{2}(z)=\left[c_{0} / c(z)\right]^{2}-\sin ^{2} \theta, p(z)$ et $p(z) / \rho(z)$ continues. A partir 
de la transformation $s^{\prime}(z)=\rho(z) / \rho_{0}$ substituant à la profondeur réelle $z$ une profondeur apparente $s$ fonction positive croissante liée à la densité du milieu de propagation, il est possible d'obtenir une nouvelle équation d'onde $p^{\prime \prime}(s)+\left[k_{0} N_{\theta}(s)\right]^{2} p(s)=0, p(s)$ et $p^{\prime}(s)$ continues, où seul apparaît un indice de réfraction $N_{\theta}(s)=\left[\rho_{0} \rho \rho(s)\right] \sqrt{ }\left[c_{0} / c(s)\right]^{2}-\sin ^{2} \theta$.

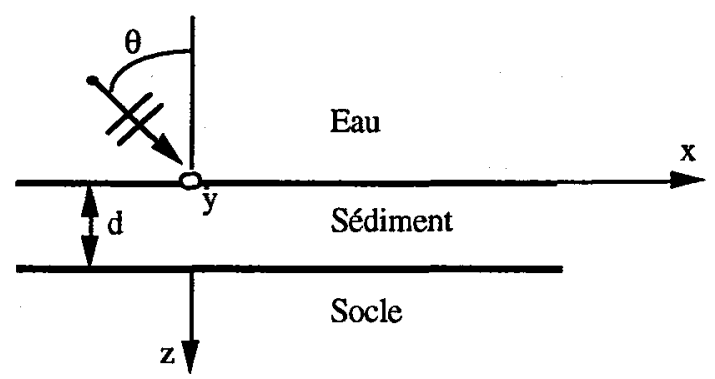

\section{Figure 1}

Cette transformation maintenant classique [4] permet d'eviter toute dérivation des paramètres acoustiques du milieu et leur substitue un indice unique purement réel à incidence sous-critique et qui décrô̂t monotoniquement dans le sédiment dans le cas standard où $\rho$ et $c$ y croissent. Ce faisant, il est clair que la connaissance de l'indice à deux incidences différentes permet d'obtenir une expression analytique simple des profils de densité et de vitesse $\rho(s)$ et $c(s)$, donc d'estimer $z(s)$, ce qui conduit aux profils vrais $\rho(z)$ et $c(z)$. Si plusieurs profils d'indice sont disponibles (e.g., par mesure à plusieurs incidences), une procédure de moindres carrés fournit des estimés des profils en fonction de $s$ et par suite en fonction de $z$ [5]. Remarquons que la détermination simultanée de $\rho(z)$ et $c(z)$ est un problème délicat quand on travaille en temps de propagation ainsi que le montre [6-8] et que le choix d'une profondeur apparente facilite cette détermination même s'il ne lève pas son caractère mal posé (e.g., des couples de profils assez différents sont associés à des coefficients de réflexion très proches). On notera enfin que le calcul de $p(s)$ pour un profil d'indice connu (problème direct) se fait rapidement et avec précision par une technique de matrices de chaîne en assimilant le milieu à un empilement de strates homogènes d'une épaisseur $\Delta s$ égale à une fraction de la longueur d'onde minimale $\lambda_{0} / \operatorname{Max}\left(N_{\theta}\right)$.

\section{Méthodes de reconstruction}

Reste à déterminer le profil d'indice à incidence donnée $\theta$. Une procédure exacte [9] nous a permis [2] de le construire à partir de la réponse impulsionnelle (la transformée de Fourier du coefficient de réflexion $R_{\theta}(\omega)$ ) par transformation de l'équation d'onde en équation de Schrödinger résolue à l'aide d'une technique de Gelfand-Levitan en "marchant dans le temps". Mais cette procédure (normalement limitée à un problème 1-D) souffre de limites théoriques (profils deux fois différentiables) et du nécessaire accès à la réponse impulsionnelle, tandis qu'elle apparaît sensible à des erreurs sur les données (tout au moins pour $\rho(z)$ et $c(z))$. En conséquence, il nous a semblé intéressant d'envisager une méthode itérative dite de Borndistordue qui permet le diagnostic de profils quelconques à partir d'un nombre restreint de données (les coefficients de réflexion à plusieurs fréquences) en tenant compte de l'information disponible au départ sur le milieu par le choix du profil d'initialisation et l'introduction de contraintes de régularité sur la solution. La façon dont nous déployons cette méthode s'inspire des travaux [10].

Nous partons de la mesure $R\left(\omega_{\mathrm{q}}\right)$ des coefficients de réflexion de l'empilement sédiment-socle $R\left(\omega_{\mathrm{q}}\right)$ (sousentendant $\theta$ ) à $Q$ fréquences discrètes $\omega_{\mathrm{f}}$ réparties selon une échelle logarithmique (nous n'utilisons pas la fréquence zéro). Nous décrivons le profil d'indice inconnu par un petit nombre $M-1$ de fonctions de base à support borné du type triangles dont les coefficients $\chi_{\mathrm{m}}$ (purement réels) deviennent alors les inconnues. Au delà d'une certaine profondeur assez grande le profil est supposé plat (milieu homogène de valeur $\chi_{\mathrm{M}}$ ). Laissant de côté les détails de la dérivation, le processus se résume ainsi. Les $Q$ coefficients de réflexion sont des sommes finies $R\left(\omega_{\mathrm{q}}\right)=\sum I_{\mathrm{m}}\left(\omega_{\mathrm{q}}\right) \chi_{\mathrm{m}}$ où les $M$ coefficients $I_{\mathrm{m}}$ sont des fonctions de la pression à $s \geq 0$. Soit l'itération $i$. On suppose obtenu un ensemble de paramètres $\left\{\chi_{\mathrm{m}}\right\}_{\mathrm{i}}$. Les pressions $\{p(s \geq 0)\}_{\mathrm{i}}$ à $\omega_{\mathrm{q}}$ s'en déduisent, donc les $\left\{I_{\mathrm{m}}\right\}_{\mathrm{i}}$ associés. Mais les coefficients de réflexion correspondants $\left\{R\left(\omega_{\mathrm{q}}\right)\right\}_{\mathrm{i}}$ peuvent différer de ceux observés, ce qui se mesure par un écart quadratique moyen supérieur à une certaine borne $\varepsilon$. Il faut donc déterminer un nouveau jeu $\left\{\chi_{m}\right\}_{i+1}$ qui rapproche les coefficients de réflexion calculés de ceux mesurés. Ce jeu n'est autre qu'une solution aux moindres carrés régularisée du système 
linéaire surdéterminé $R\left(\omega_{\mathrm{q}}\right)=\Sigma I_{\mathrm{m}}\left(\omega_{\mathrm{g}}\right) \chi_{\mathrm{m}}$, solution qui privilégie les fréquences basses (par multiplication de la ligne $q$ par le coefficient $\omega_{q} / \omega_{1}$ ) et les profils doux (en complétant le systeme par $M$ équations de forme générique $\delta\left[\chi_{\mathrm{m}+1}-2 \chi_{\mathrm{m}}+\chi_{\mathrm{m}-1}\right]=0$ où $\delta$ traduit le poids accordé à cette "douceur"). Cette solution obtenue, elle produit des coefficients de réflexion qui satisfont ou non le critère écart inférieur à $\varepsilon$. Si oui, le processus s' arrête avec succès. Si non, il se poursuit à l'itération $i+1$.

Ainsi le problème inverse non linéaire de la recherche du profil d'indice est remplacée par une succession de problèmes inverses linéaires formulés dans une hypothèse de petites variations (une faible variation des profils est associé à une variation proportionnelle des champs) et convenablement régularisés, ce qui, s'il y a convergence (elle n'est pas assurée), fournit une solution au problème initial. Il est cependant possible de construire une solution approchée qui prend en compte au moins partiellement le caractère non linéaire de l'inversion et ne se limite donc pas à l'application d'une approximation de Born (ce qui nous conduit a l'impédiographie quand on travaille en temps de propagation). Une telle solution a été développée pour le diagnostic d'un profil de permittivité d'une lame diélectrique inhomogène [11] (et mise en perspective en [10]). Ici, elle revient à exprimer que, de manière approchée, l'écart $N_{\theta}(s)^{2}-N_{\theta}(s<0)^{2}$ est liée à la réponse du milieu à un échelon (la transformée de Fourier de $R(\omega) / \omega)$ sur laquelle on applique une correction d'amplitude puis une correction de position. Cette approche nécessite évidemment la disposition de $R$ sur une plage de fréquences assez large et au moins sa bonne estimation à la fréquence zéro, mais les résultats obtenus à l'origine et confirmés ici montrent sa validité à faible coût de calcul (en comparaison de l'approche précédente) pour une large gamme de profils non nécessairement de variations douces.

\section{Résultats numériques}

Les résultats présentés ont trait à la reconstruction sur une profondeur de $12 \mathrm{~m}$ d'une couche inhomogène de $10 \mathrm{~m}$ d'épaisseur et de profils de vitesse et densité linéaires continuée par le socle homogène, les réponses du milieu $R$ - bruitées ou non - étant simulées à deux incidences $\left(0^{\circ}\right.$ et $\left.20^{\circ}\right)$.

Ces résultats et ceux obtenus par ailleurs font apparaître que les reconstructions des profils $N_{\theta}(s)$ sont excellentes que ce soit sans ou avec bruit, mais qu'il n'en est pas de même avec $c(z)$ et $\rho(z)$. En effet ces derniers profils sont très sensibles aux erreurs sur les données (notamment à celles introduites sur la phase du coefficient de réflexion), sans qu'une amélioration notable soit constatée par rapport à l'approche exacte (Gelfand-Levitan), et même, dans le cas de la solution approchée, avec une nette détérioration.

En ce qui concerne plus particulièrement la méthode de Born-distordue, nous remarquons principalement que sa convergence est en règle générale assurée, sous réserve que l'on impose comme profil reconstruit à une itération donnée, non pas le profil juste obtenu, mais sa moyenne avec le profil précédent. On note aussi que l'écart quadratique moyen entre coefficient mesuré $R$ et calculé $R$ ne décrô̂t pas monotoniquement, ce qui ne facilite pas le choix d'arrêter ou non le processus itératif, et que des coefficients distinctement différents aux fréquences élevées (ici au delà d'une centaine de $100 \mathrm{~Hz}$ ) peuvent être associés à des indices $N_{\theta}$ quasi identiques, ce qui correspond au caractère mal posé de la recherche simultanée de $c$ et $\rho$. Rappelons enfin qu'un des avantages de la méthode se confirme, le fait qu'il suffit pour obtenir de bons résultats de disposer d'un petit nombre de fréquences d'observation placées dans une bande de fréquence assez restreinte (ici comprise entre quelques dizaines et quelques centaines de $\mathrm{Hz}$ ).

\section{Références}

[1] BREKHOVSKIKH, L. M. et LYSANOV, Y., Fundamentals of Ocean Acoustics, Springer (1982).

[2] BOHBOT, R. et al., Proc. IMACS'91, Dublin (1991) 477.

[3] LAMBERT, M. et al., Proc. Col. Acoust. Sous-Marine et Ultrasons, LMA Marseille (1991) 133.

[4] GODIN, O. A., Wave Motion 7 (1985) 515.

[5] SANTOSA, F. et SYMES, W. W., Geophys. J. R. astr. Soc. 81 (1985) 175.

[6] HOOSHYAR, M. A. et RAZAVY, M., J. Acoust. Soc. Am. 73 (1983) 19.

[7] HOWARD, M. S., Geophys. 48 (1983) 163.

[8] CARRION, P. M., J. Acoust. Soc. Am. 77 (1985) 1370.

[9] COEN, S., Geophys. 46 (1981) 1244.

[10] TIJHUIS, A. G., Wave Motion 11 (1989) 151.

[11] TABBARA, W., IEEE Trans. Antennas Propagat. AP-27 (1979) 241. 

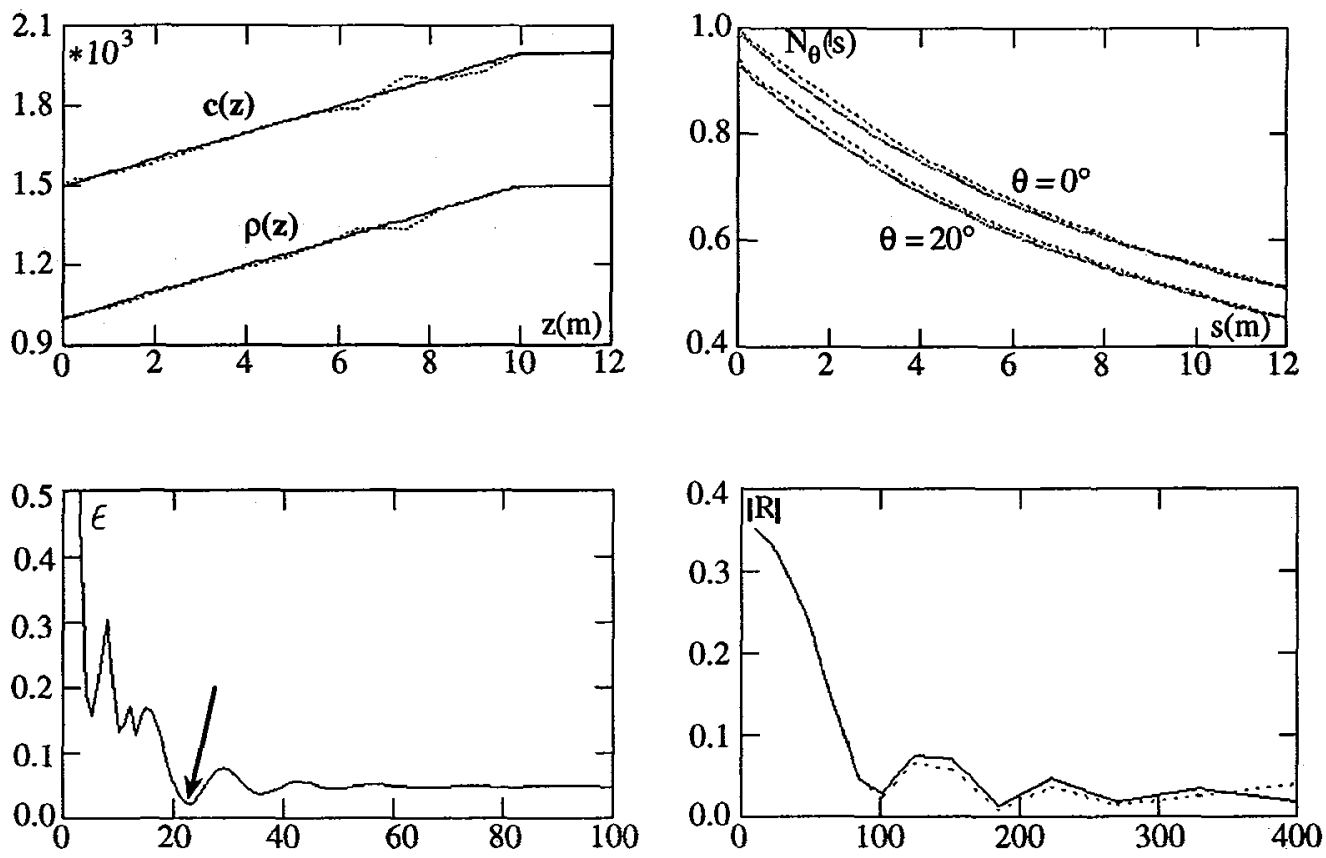

Fig. 2 : Méthode itérative de Born-distordue. Sont utilisés des coefficients de réflexion exacts calculés à 20 fréquences $\omega_{\mathrm{q}}$ réparties de manière logarithmique entre 10 et $400 \mathrm{~Hz}$. Le processus implique un moyennage des profils (voir texte), une pondération linéaire en fréquence et une régularisation de faible importance $(\delta=$ $10^{-3}$ ). Les paramètres de l'eau $c_{0}=1500 \mathrm{~m} / \mathrm{s}$ et $\rho_{0}=1$ servent à l'initialisation.

(a) et (b) : Vitesses (en $\mathrm{m} / \mathrm{s})$, densités et indices exacts (- - et reconstruits (- - ). (c) Evolution de l'écart quadratique moyen $E=\sum\left|\left(R_{\mathrm{i}}-R\right)\left(\omega_{\mathrm{q}}\right)\right|^{2} / \sum\left|R_{\mathrm{i}}\left(\omega_{\mathrm{q}}\right)\right|^{2}$ entre coefficient de réflexion mesuré $R$ et reconstruit $R_{\mathrm{i}}$ en fonction du nombre $i$ d'itérations. La valeur minimale (à laquelle correspond les profils reconstruits donnés en (a), (b) et (d)) est indiquée par la flèche. (d) Module du coefficient de réflexion exact (-) et reconstruit (- - ) en fonction de la fréquence $(\mathrm{en} \mathrm{Hz})$.
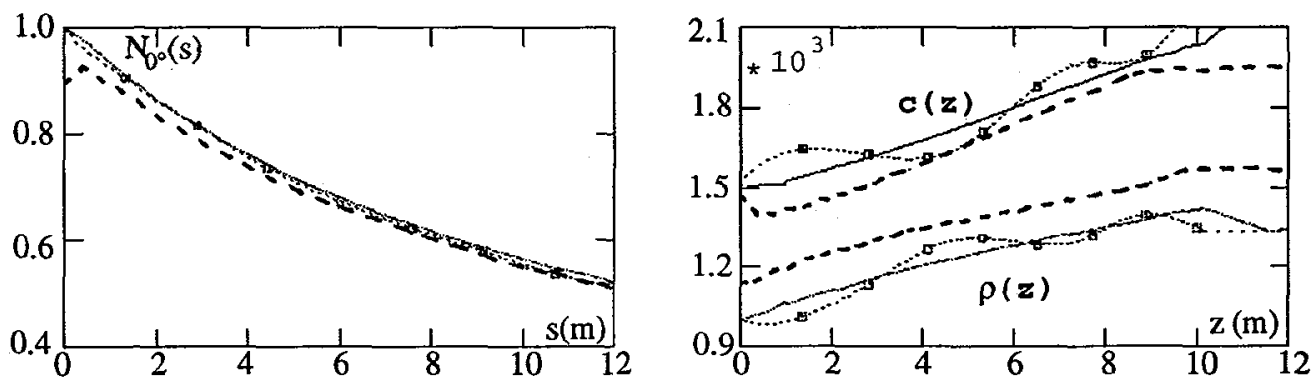

Fig. 3 : Performances des diverses méthodes en présence de données bruitées (mêmes profils que Fig. 2). Celles-ci sont obtenues en affectant, simultanément et de manière indépendante, au module et à la phase du coefficient de réflexion $R$ des valeurs de la forme $a(1+b)$ où $a$ est la valeur exacte et où $b$ est aléatoire uniforme de moyenne nulle et compris entre \pm 0.01 . Sont présentés les vitesses (en $\mathrm{m} / \mathrm{s}$ ), densités et indice (à $\theta=0^{\circ}$ ) reconstruits par les méthodes de Born-distordue $(\square---\square)$, approchée (- - ) et de GelfandLevitan $(\longrightarrow)$. Les indices reconstruits sont très proches de l'indice exact. 